\title{
Pérdida de visión por diabetes: detección y tratamiento
}

\author{
Stéphanie Thébault
}

\section{Resumen}

La retinopatía diabética (RD), enfermedad de la retina comúnmente clasificada como una complicación vascular de la diabetes, es ahora reconocida como una afección que implica tanto a los vasos como a las neuronas (neurovascular). Muchas evidencias apuntan a que esta neuropatía sensorial resulta de la ruptura de la unidad neurovascular. A pesar de ello, las terapias actuales se centran en limitar las anomalías vasculares, incluyendo la formación de vasos sanguíneos irregulares y la hinchazón en la parte central de la retina (conocida como edema macular). Con base en que la ruptura de la unidad neurovascular es un evento temprano en la RD, muchos esfuerzos científicos se centran en encontrar los mecanismos que se vinculan con su ruptura, para lograr el desarrollo de nuevos tratamientos en etapas tempranas. Sin embargo, también hace falta desarrollar y validar nuevos marcadores tempranos de la función visual para reducir la duración de los ensayos clínicos y acelerar la entrega de nuevos tratamientos contra la RD para la población en general. En este trabajo, revisamos qué es la unidad neurovascular retiniana y las alteraciones tempranas que la misma sufre en condiciones de diabetes, y examinamos a detalle la necesidad de generar criterios de valoración temprana de la función visual para los futuros ensayos clínicos que evalúan los nuevos tratamientos para la RD.

Palabras clave: retinopatía diabética, visión, unidad neurovascular, criterios de valoración funcional temprana, terapias.

\section{DIABETIC RETINOPATHY: EARLY DETECTION}

\begin{abstract}
Diabetic retinopathy (DR), commonly classified as a vascular complication of diabetes, is now recognized as a neurovascular affliction. A comprehensive set of evidence points to the fact that DR is a sensory neuropathy resulting from the neurovascular unit rupture, and that neuronal death is an early event in the pathogenesis of DR. However, current therapies for DR only focus on limiting the vascular alterations, including abnormal formation of new blood vessels and macular edema. Based on this information, much scientists' efforts are about elucidating the mechanisms underlying both neurodegeneration and neurovascular unit rupture to develop new treatments for initial stages of DR. The identification and validation of early markers of visual function is also required to reduce the duration of clinical trials and to accelerate the delivery of new treatments for DR to the general population. In this article, we describe
\end{abstract}

Dol: http://doi.org/10.22201/codeic.16076079e.2019.v20n3.a1 
the neurovascular unit of the retina and examine how the retina is altered in early stages of diabetes. We also comment on how early functional endpoint of visual function would impact future clinical trials that aim to assess novel therapeutic options for DR.

Keywords: diabetic retinopathy, vision, neurovascular unit, early functional endpoint, therapies.

\section{Stéphanie Thébault}

stephaniethebault@gmail.com

Investigadora en el Instituto de Neurobiología de la Universidad Nacional Autónoma de México, Departamento de Neurobiología Celular y Molecular, Laboratorio de Neurobiología Molecular y Celular. Es miembro del Sistema Nacional de Investigadores, nivel 2 y PRIDE C. Dra. en Ciencias de la Vida y de la Salud por la universidad de Ciencias y Tecnologías de Lille, Francia. Sus temas de investigación son el estudio del papel de canales iónicos y de la actividad eléctrica en la retina y sus implicaciones en diversas patologías retinianas asociadas con inflamación crónica, incluyendo la RD. 


\section{¿Qué es la retinopatía diabética?}

La retinopatía diabética (RD) es la principal causa de ceguera en personas laboralmente activas $y$, tradicionalmente, se ha considerado como una complicación de la diabetes que afecta los vasos sanguíneos (complicación vascular).

Al clasificar esta enfermedad clínicamente, se toman en cuenta sólo los cambios estructurales en los vasos de la retina (Aiello, 2003; Wilkinson et al., 2003), pues durante la exploración del fondo de ojo (oftalmoscopia) la formación excesiva de nuevos vasos sanguíneos anómalos es visible -ya que la retina es transparente-, y ese daño vascular coincide con la pérdida de visión.

Fue hasta la década de los sesenta cuando Wolter (1961) y Bloodworth (1962) identificaron que existe un componente neuronal en la retina. A partir de un estudio con diabéticos post mortem, descubrieron neuronas en proceso de degradación en la retina. Desde entonces, se ha acumulado mucha evidencia sobre la participación de las neuronas en la RD (Cunha-Vaz, 2012). Actualmente se realizan ensayos que exploran el efecto de varias terapias diseñadas para revertir el daño neuronal en la RD (Cunha-Vaz, 2012). Sin embargo, esta enfermedad no se atiende si no se presentan cambios vasculares visibles, aunque las lesiones en las neuronas ocurren y evolucionan al mismo tiempo -0 hasta antes - que las lesiones vasculares. Eso se debe a que no existe un punto de control funcional temprano para detectar la enfermedad.

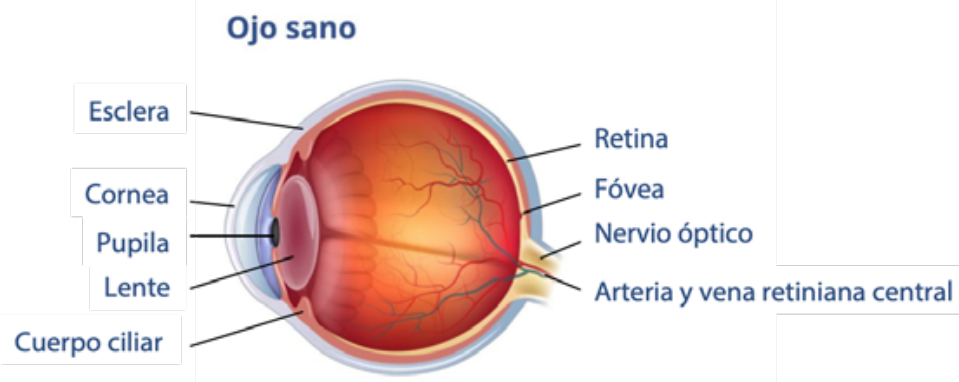

Figura 1. Se muestra la composición de la retina y las alteraciones vasculares en la RD.

\section{Retinopatía diabética}

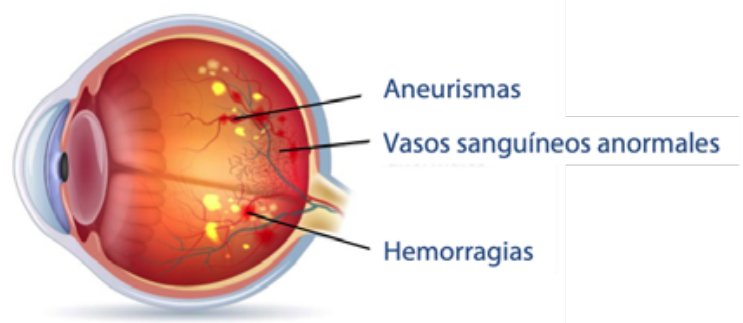

Con el fin de reducir la pérdida de la visión por esta enfermedad, se ha enfatizado mucho el componente vascular y los tratamientos se han dirigido hacia los vasos 
sanguíneos. De esta manera, se ha considerado muy poco la contribución de la retina neural en este proceso.

Para tratar la RD, desde hace cinco décadas, se ha empleado la fotocoagulación panretiniana, una cirugía ocular que utiliza un láser en áreas extensas de la retina que se centra en tratar los microaneurismas, que son pequeñas dilataciones en forma de saco en los vasos sanguíneos, con escape en la retina. Sin embargo, los efectos de este tratamiento son indirectos, pues es altamente eficaz para preservar la agudeza visual central (Group, 1976), pero reduce el campo de visión periférica (Doft y Blankenship, 1982; Early Treatment Diabetic Retinopathy Study Research Group, 1991; Pahor, 1998) y la visión nocturna (Pender et al., 1981; Russell et al., 1985; Seiberth et al., 1987). Por lo tanto, se puede considerar que la fotocoagulación panretiniana es similar a una amputación, en el sentido que la retina periférica se sacrifica para que la visión central pueda ser preservada. Los efectos secundarios de esta cirugía con láser han promovido investigaciones para desarrollar estrategias farmacológicas para mejorar la RD. Actualmente, veintinueve fármacos para tratar la RD se encuentran en ensayos clínicos fase ॥ (doce), fase ॥ (nueve) y fase ıv (ocho) (como se puede ver aquí).

Antes de que llegarán las inyecciones intraoculares, el edema macular secundario a la diabetes se trataba también con láser focal en el centro de la mácula. El edema macular diabético corresponde a la hinchazón de la mácula, que es la zona del ojo responsable de la visión de los detalles. Actualmente se trata con inyecciones intraoculares de agentes que controlan los vasos sanguíneos (anti-VEGF), los cuales son una gran avance en comparación con la fotocoagulación con láser focal ya que mejoran la agudeza visual y ralentizan la progresión de las lesiones vasculares (Nguyen et al., 2009). Sin embargo, estos agentes sólo mejoran la agudeza visual de entre 30 y $50 \%$ de los pacientes afectados (Mitchell et al., 2011; Nguyen et al., 2012 y 2009), lo que sugiere que otros fenómenos intervienen en la patogénesis de la RD, además de la sobrerregulación de VEGF.

Por todo lo anterior, queda claro que muchas personas se verían beneficiadas por tratamientos que se dirigen a atacar la progresión de la RD en sus etapas iniciales (Simo et al., 2012). La obtención de dichas terapias depende del desarrollo de marcadores tempranos de la función visual, la cual, a su vez, depende de la integridad de la unidad neurovascular retiniana.

\section{La unidad neurovascular de la retina}

En todo el sistema nervioso, las neuronas, las células gliales — que son células del tejido nervioso que complementan la actividad neuronal-, así como los vasos sanguíneos se organizan en unidades neurovasculares. El estrecho contacto físico entre estos diferentes tipos de células permite una integración funcional que media las respuestas de adaptación a diversas condiciones. Las unidades neurovasculares coordinan la demanda energética (metabólica), la actividad de 
Figura 2. Las neuronas (células ganglionares, amacrinas, bipolares, horizontales, conos y bastones), la glía (células de Müller, astrocitos y células de la microglia) y las células vasculares comprenden la unidad neurovascular de la retina. La gran cantidad de conexiones fisiológicas y anatómicas entre estas células permite la visión. las sinapsis, la entrega de sangre y la eliminación de residuos como el glutamato, el óxido nítrico, la adenosina, los metabolitos del ácido araquidónico y varios ácidos (Kur et al., 2012; Metea y Newman, 2007).

En términos generales, la actividad de las neuronas depende de los vasos sanguíneos, ya que éstos entregan nutrientes y eliminan los productos de desecho de los tejidos. Además, intervienen las células gliales o de la glia, de las cuales existen varios tipos: los astrocitos, la microglia y un tipo específico de la retina: la glia de Müller. Las células de Müller coordinan las respuestas vasculares para satisfacer la demanda metabólica de las neuronas, el intercambio de metabolitos, el reciclaje de neurotransmisores como el glutamato, y también ayudan a establecer los entornos químicos afuera de las neuronas, para que se den el potencial de membrana y la actividad eléctrica adecuados. Los astrocitos hacen contacto con los vasos sanguíneos para mantener su forma y función (Petzold y Murthy, 2011). Las células de la microglia monitorean la actividad celular local y la actividad sináptica (Schafer et al., 2012), y sirven para deshacerse de células muertas. La actividad coordinada de todas estas células (neuronas, células gliales y vasculares) es esencial para la visión normal (ver figura 1).

\section{Los componentes de la unidad neurovascular en la retina}

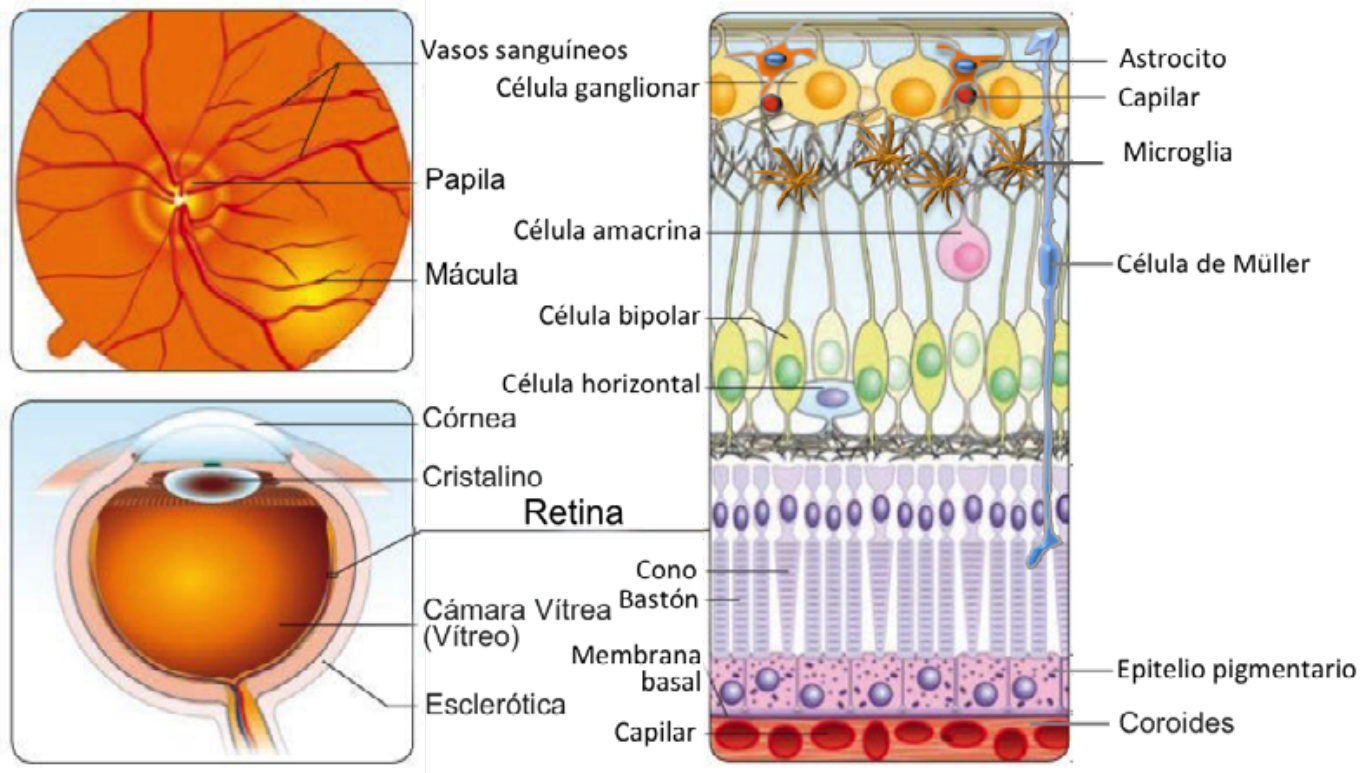

Aunque muchos detalles sobre las conexiones entre las células que forman la unidad neurovascular de la retina quedan por ser determinados, se ha podido caracterizar las relaciones físicas entre ellas. Las neuronas se comunican a través de sinapsis químicas, que utilizan neurotransmisores como glutamato, dopamina, ácido gamma aminobutírico o GABA, acetilcolina o glicina; y a través de sinapsis eléctricas (uniones comunicantes) que están conformadas por 
Figura 3. Alteraciones sub/ preclínicas de la retinopatía diabética (RD).

ensambles de proteínas de tipo conexina, que permiten el flujo de iones entre células adyacentes (Volgyi et al., 2013). Las células vasculares se comunican entre ellas mediante uniones estrechas (Runkle y Antonetti, 2011). Estas conexiones complejas revelan por qué los primeros anatomistas que las estudiaron llamaron a esa red de células retina, que en latín medieval significa "túnica como una red".

\section{Las alteraciones tempranas de la retina en la diabetes}

El curso temporal preciso y la serie de eventos que llevan a la aparición de la RD quedan por ser definidos con claridad. Sin embargo, las evaluaciones de los pacientes con diabetes sin retinopatía indican que el daño de la estructura y función de la parte neural de la retina precede las lesiones vasculares clínicamente observables. Éstas, tradicionalmente, incluyen los microaneurismas, las hemorragias (sangrados) y los exudados lipídicos (depósitos). Cabe señalar que se observan modificaciones similares de la unidad neurovascular en el cerebro de pacientes con otras enfermedades neurodegenerativas como Alzheimer y Parkinson (del Zoppo, 2010; Zlokovic, 2008). Además, se sabe que

\section{Retinopatía Diabética (RD)}

Fase pre/subclínica

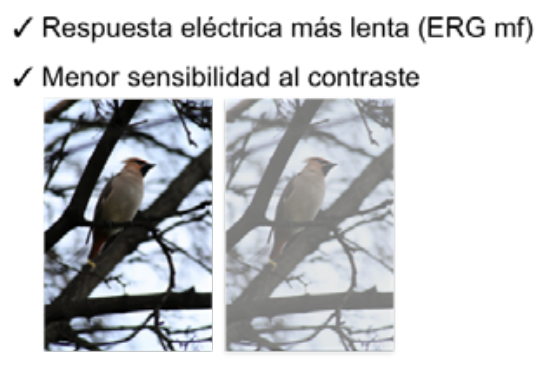

$\checkmark$ Menor adaptación a la oscuridad

$\checkmark$

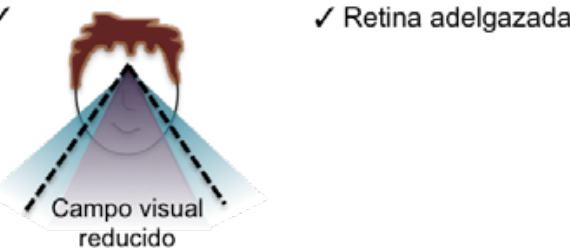

$\checkmark$
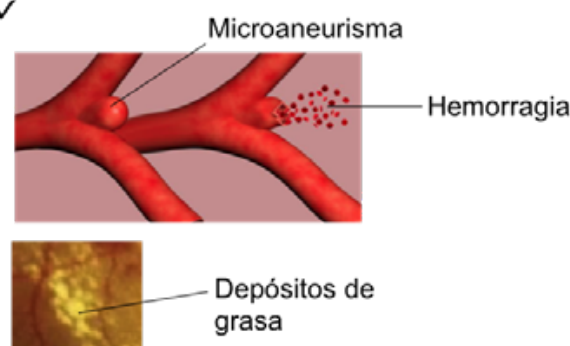

en pacientes con diabetes tipo 1 o tipo 2 que todavía no presentan signos visibles de retinopatía estas alteraciones se manifiestan en forma de una incapacidad de contraer y dilatar sus vasos sanguíneos en respuesta a respirar oxígeno al 100\% o recibir luz parpadeante, respectivamente (Bek et al. 2008; Garhofer et al., 2004; Lasta et al., 2013; Lott et al., 2012). Estos cambios parecen reflejar el deterioro precoz de los mecanismos reguladores que normalmente existen en la unidad neurovascular de la retina, $y$ no el ser alteraciones aisladas en los vasos sanguíneos (Antonetti et al., 2012).

Se han reportado más alteraciones en la fase preclínica de la RD, que incluyen una respuesta más lenta en el electrorretinograma multifocal (mfERG, por sus siglas en inglés), la cual predice la aparición de lesiones vasculares (Harrison et al., 2011; Ng et al., 2008), una sensibilidad reducida al contraste luminoso y a la adaptación en la oscuridad (Greenstein et al., 1993) y una disminución en el campo visual (Jackson et al., 2012; Parravano et al., 2008). Asimismo, se ha medido una reducción en el espesor de la retina interna en la fase preclínica (Park et al., 2011; van Dijk et al., 2010), como se puede ver en la figura 2 . 
En conjunto, todas estas observaciones muestran que la diabetes tiene efectos deletéreos tempranos sobre la estructura y función de la unidad neurovascular retiniana, y también indican que reconocer las alteraciones subclínicas en la función de la unidad neurovascular puede ayudar a identificar a las personas en riesgo de pérdida de visión.

Algunas de estas alteraciones pueden ser adaptaciones fisiológicas a la condición de diabetes, las cuales pretenden mantener la retina viva y funcional, para conservar la visión. Se considera que las características clínicas de la RD aparecen cuando estas adaptaciones ya no logran mantener viable la retina. Asimismo, cabe la posibilidad de que las alteraciones anteriormente referidas no sean nada más adaptaciones fisiológicas de esta condición, sino que también sean cambios que ocurren en respuesta a la hipertensión o la inflamación que acompañan la diabetes (Antonetti et al., 2006).

Además, cabe mencionar que en las fases clínicas de la RD, la ruptura progresiva de la unidad neurovascular y la pérdida de la capacidad de autorregulación resultan en tortuosidades y dilatación de las venas, que conllevan a la aparición del edema macular y de la RD proliferativa (Kristinsson et al., 1997; Kylstra et al., 1986; Sinclair et al., 1982). Por lo tanto, se puede considerar que las alteraciones de unidad neurovascular retiniana forman parte de un espectro más amplio de efectos sobre el sistema nervioso en relación con la diabetes, que incluyen a las neuropatías sensoriales y autónomas, así como al deterioro cognitivo (Reijmer et al., 2013; Umegaki, 2012).

Los factores oculares y circulantes que contribuyen al desarrollo y progresión de la RD han sido complejos y difíciles de aproximar, debido a la falta de modelos animales que recapitulen completamente la patología humana. No obstante, la comprensión actual de su patogénesis se debe al estudio de modelos en roedores, en paralelo con el análisis de pacientes de una manera integrativa (Gardner et al., 2011). Gracias a ello, se sabe que entre los factores que participan en el desarrolloy la progresión de la RD se encuentran las alteraciones metabólicas, incluyendo la hiperglucemia, reducción de la señalización a través del receptor a insulina, estrés oxidativo, formación de productos finales de glicación avanzada, alteraciones lipídicas, entre otras; la inflamación, principalmente caracterizada por niveles aumentados de citocinas pro-inflamatorias; la toxicidad asociada al glutamato; y los niveles reducidos de factores neuroprotectores naturalmente presentes en la retina.

\section{Sobre los criterios de valoración temprana de la función visual en la diabetes}

La alta frecuencia de RD al momento del diagnóstico de la diabetes tipo 2 se debe a que esta enfermedad comienza entre cuatro y siete años antes del diagnóstico clínico. A los 10 años de diabetes tipo 2, entre 35 y 40\% de los pacientes presentan RD, llegando a 80\% a los 20 años de evolución. La RD provoca poca sintomatología 
en sus etapas iniciales y no afecta la visión hasta etapas muy avanzadas. Esto hace que, con frecuencia, el diagnóstico, que se basa en la detección de anomalías vasculares por inspección visual, sea tardío y que, al momento del diagnóstico, en $10 \%$ de los casos se detecten complicaciones serias, como hemorragia vítrea, desprendimiento de retina traccional o edema macular (Cohen y Gardner, 2016). Por lo tanto, es necesario establecer criterios de valoración temprana, para detectar la RD, mediante puntos de control funcional tempranos (ver figura 3).

CRITERIOS DE VALORACIÓN

TEMPRANA

de la función retiniana

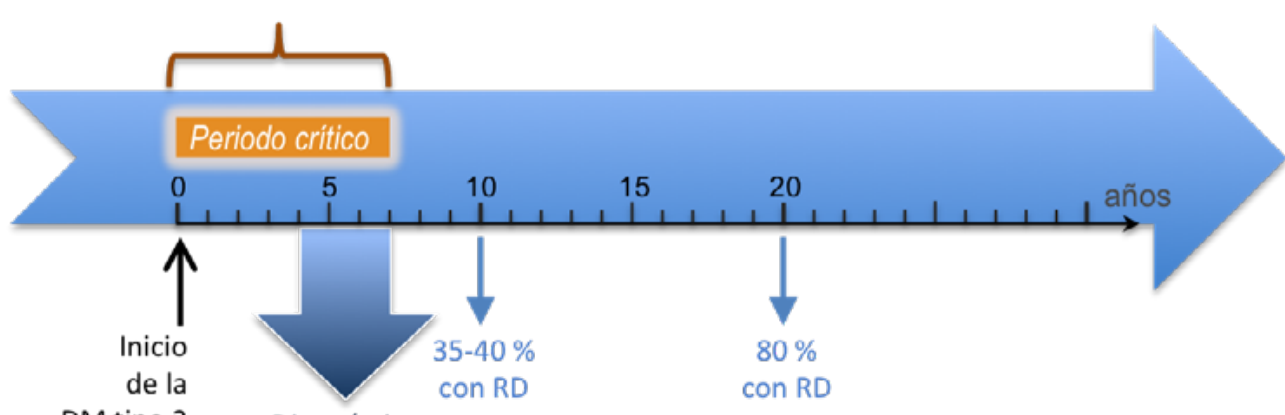

Figura 4. Curso temporal de la aparición de la retinopatía diabética (RD).

De igual manera, el uso de criterios de valoración para la visión alternos podría acortar la duración de los ensayos clínicos y acelerar el desarrollo de nuevas terapias contra la RD (Stem y Gardner, 2013). Para ello, el consorcio europeo para el grupo de estudio de tratamiento temprano de RD (EUROCONDOR) ha estado usando el tiempo implícito del mfERG como su principal criterio de evaluación para medir la disfunción de las neuronas en la retina. No obstante, otros criterios de valoración funcional, como el mfERG, necesitan ser desarrollados y estandarizados para evaluar la eficacia de las nuevas estrategias neuroprotectoras para la RD. En efecto, se ha propuesto el uso de agentes que reducen la inflamación, restauran el receptor de la insulina en la retina, inhiben receptores a glutamato o bloquean la muerte celular programada, los cuales son promisorios como nuevas terapias para tratar la RD en etapas tempranas. Sin embargo, hace falta evaluar, en estudios clínicos, estas opciones terapéuticas mediante criterios de valoración temprana de la función retiniana antes de que dichos agentes puedan ser utilizados para tratar el número, siempre creciente, de pacientes con RD.

El hecho de que sólo 7\% de las personas con diabetes tipo 2 (Saydah et al., 2004) y un tercio de los niños con diabetes tipo 1 cumplan con los objetivos terapéuticos para los niveles de hemoglobina glicosilada (HbA1c) en sangre de la Asociación Americana de Diabetes y de la Sociedad Internacional para los lineamientos clínicos sobre la diabetes en infantes y adolescentes (Wood et al., 2013) revela lo difícil y complejo que es prevenir y tratar la RD. Es por ello 
que la disponibilidad de métodos más cuantitativos y sensibles para la detección preclínica de los daños de la unidad neurovascular en la retina será crítica en un futuro próximo (Jackson et al., 2012; Lott et al., 2012).

\section{Conclusiones}

La RD se entiende ahora como una neuropatía sensorial, similar a otras que afectan a las personas con diabetes. Las terapias actuales se centran en mitigar las secuelas vasculares avanzadas de la RD. Hoy en día se están probando agentes con propiedades neuroprotectoras para determinar su capacidad de disminuir la pérdida visual progresiva que se asocia a la diabetes. Los mecanismos moleculares implicados en la degradación neuronal asociada a la RD son complejos e incluyen, probablemente, una combinación de factores oculares, tales como niveles excesivos de estrés oxidativo y de inflamación, de factores neuroprotectores naturales, toxicidad relacionada con el glutamato y con factores circulantes como la deficiencia a la insulina, hiperglucemia y dislipidemias. El desarrollo de nuevas terapias requiere identificar criterios de valoración funcional temprana de la función retiniana, que reflejen con precisión la función visual, y que puedan utilizarse como nuevos marcadores de la agudeza visual.

\section{Referencias}

* Aiello, L. M. (2003). Perspectives on diabetic retinopathy. American Journal of Ophthalmology, 136(1), 122-135. Dol: https://doi.org/10.1016/S0002-9394(03)00219-8.

- Antonetti, D. A., Barber, A. J., Bronson, S. K., Freeman, W. M., Gardner, T. W., Jefferson, L. S., ... Group, J. D. R. C. (2006). Diabetic retinopathy: seeing beyond glucose-induced microvascular disease. Diabetes, 55(9), 2401-2411. Dol: https://doi. org/10.2337/db05-1635.

- Antonetti, D. A., Klein, R. y Gardner, T. W. (2012). Diabetic retinopathy. New England Journal of Medecine, 366(13), 1227-1239. Dol: https://doi.org/10.1056/NEJMra1005073.

* Bek, T., Hajari, J. y Jeppesen, P. (2008). Interaction between flicker-induced vasodilatation and pressure autoregulation in early retinopathy of type 2 diabetes. Graefes Archive Clinical Experimental Ophthalmology, 246(5), 763-769. DOI: https://doi. org/10.1007/s00417-008-0766-y.

Bloodworth, J. M. J. (1962). Diabetic retinopathy. Diabetes, 11, 1-22.

* Cohen, S. R.y Gardner, T.W.(2016). Diabetic Retinopathy and Diabetic Macular Edema. Developemts in Ophthalmology, 55, 137-146. Dol: https://doi.org/10.1159/000438970.

* Cunha-Vaz,J. (2012). Neurodegeneration as an eraly event in the pathogenesis of Diabetic Retinopathy: A multicentric, prospective, phase II-II, randomised controlled trial to asses the efficacy of neuroprotective drugs administered topically to prevent or arrest Diabetic Retinopathy. EUROCONDOR-EU FP7 Project. Acta Ophtalmologica, 90(249), 0.

delZoppo, G.J.(2010). The neurovascularunitinthe setting ofstroke.Journal of Internal 
Medecine, 267(2), 156-171. Dol: https://doi.org/10.1111/j.1365-2796.2009.02199.x.

- Doft, B. H. y Blankenship, G. W. (1982). Single versus multiple treatment sessions of argon laser panretinal photocoagulation for proliferative diabetic retinopathy. Ophthalmology, 89(7), 772-779.

* Early Treatment Diabetic Retinopathy Study Research Group (1991). Early photocoagulation for diabetic retinopathy. ETDRS report number 9. Ophthalmology, 98(5 Suppl), 766-785. Dol: https://doi.org/10.1016/S0161-6420(13)38011-7.

* Gardner, T. W., Abcouwer, S. F., Barber, A. J. y Jackson, G. R. (2011). An integrated approach to diabetic retinopathy research. Archive Ophthalmology, 129(2), 230 235. Dol: https://doi.org/10.1001/archophthalmol.2010.362.

* Garhofer, G., Zawinka, C., Resch, H., Kothy, P., Schmetterer, L. y Dorner, G. T. (2004). Reduced response of retinal vessel diameters to flicker stimulation in patients with diabetes. British Journal of Ophthalmology, 88(7), 887-891. Dol: https://doi. org/10.1136/bjo.2003.033548.

Greenstein, V. C., Thomas, S. R., Blaustein, H., Koenig, K. y Carr, R. E. (1993). Effects of early diabetic retinopathy on rod system sensitivity. Optometry and Vision Science, 70(1), 18-23.

- Group, T. D. R. S. R. (1976). Preliminary report on effects of photocoagulation therapy. American Journal of Ophtalmology, 81(4), 383-396.

Harrison, W. W., Bearse, M. A., Jr., Ng, J. S., Jewell, N. P., Barez, S., Burger, D., Adams, A. J. (2011). Multifocal electroretinograms predict onset of diabetic retinopathy in adult patients with diabetes. Investigation in Ophthalmology and Vision Science, 52(2), 772-777. Dol: https://doi.org/10.1167/iovs.10-5931.

Jackson, G. R., Scott, I. U., Quillen, D. A., Walter, L. E. y Gardner, T. W. (2012). Inner retinal visual dysfunction is a sensitive marker of non-proliferative diabetic retinopathy. British Journal of Ophthalmology, 96(5), 699-703. Dol: https://doi. org/10.1136/bjophthalmol-2011-300467.

* Kristinsson, J. K., Gottfredsdottir, M. S. y Stefansson, E. (1997). Retinal vessel dilatation and elongation precedes diabetic macular oedema. British Journal of Ophthalmology, 81(4), 274-278.

* Kur, J., Newman, E. A. y Chan-Ling, T. (2012). Cellular and physiological mechanisms underlying blood flow regulation in the retina and choroid in health and disease. Progress in Retina and Eye Research, 31(5), 377-406. Dol: https://doi.org/10.1016/j. preteyeres.2012.04.004.

* Kylstra, J. A., Wierzbicki, T., Wolbarsht, M. L., Landers, M. B., 3rd y Stefansson, E. (1986). The relationship between retinal vessel tortuosity, diameter, and transmural pressure. Graefes Archive Clinical and Experimental Ophthalmology, 224(5), 477-480.

- Lasta, M., Pemp, B., Schmidl, D., Boltz, A., Kaya, S., Palkovits, S., . . Schmetterer, L. (2013). Neurovascular dysfunction precedes neural dysfunction in the retina 
of patients with type 1 diabetes. Investigation in Ophthalmology and Vision Science, 54(1), 842-847. Dol: https://doi.org/10.1167/iovs.12-10873.

- Lott, M. E., Slocomb, J. E., Shivkumar, V., Smith, B., Gabbay, R. A., Quillen, D., Bettermann, K. (2012). Comparison of retinal vasodilator and constrictor responses in type 2 diabetes. Acta Ophthalmologica, 90(6), e434-441. DOI: https:// doi.org/10.1111/j.1755-3768.2012.02445.x.

- Metea, M. R. y Newman, E. A. (2007). Signalling within the neurovascular unit in the mammalian retina. Experimental Physiology, 92(4), 635-640. Dol: https://doi. org/10.1113/expphysiol.2006.036376.

* Mitchell, P., Bandello, F., Schmidt-Erfurth, U., Lang, G. E., Massin, P., Schlingemann, R. O., RESTORE study group (2011). The RESTORE study: ranibizumab monotherapy or combined with laser versus laser monotherapy for diabetic macular edema. Ophthalmology, 118(4), 615-625. Dol: https://doi.org/10.1016/j.ophtha.2011.01.031.

* Ng, J. S., Bearse, M. A., Jr., Schneck, M. E., Barez, S. y Adams, A. J. (2008). Local diabetic retinopathy prediction by multifocal ERG delays over 3 years. Investigation in Ophthalmology and Vision Science, 49(4), 1622-1628. Dol: https://doi.org/10.1167/ iovs.07-1157.

* Nguyen, Q. D., Brown, D. M., Marcus, D. M., Boyer, D. S., Patel, S., Feiner, L., . . . Group, R. R. (2012). Ranibizumab for diabetic macular edema: results from 2 phase III randomized trials: RISE and RIDE. Ophthalmology, 119(4), 789-801. DOI: https://doi.org/10.1016/j.ophtha.2011.12.039.

* Nguyen, Q. D., Shah, S. M., Heier, J. S., Do, D. V., Lim, J., Boyer, D., ... Group, R.-S. (2009). Primary End Point (Six Months) Results of the Ranibizumab for Edema of the mAcula in diabetes (READ-2) study. Ophthalmology, 116(11), 2175-2181 e2171. DOI: https://doi.org/10.1016/j.ophtha.2009.04.023.

* Pahor, D. (1998). Visual field loss after argon laser panretinal photocoagulation in diabetic retinopathy: full- versus mild-scatter coagulation. International Ophthalmology, 22(5), 313-319.

Park, H. Y., Kim, I. T. y Park, C. K. (2011). Early diabetic changes in the nerve fibre layer at the macula detected by spectral domain optical coherence tomography. British Journal of Ophthalmology, 95(9), 1223-1228. Dol: https://doi.org/10.1136/ bjo.2010.191841.

* Parravano, M., Oddone, F., Mineo, D., Centofanti, M., Borboni, P., Lauro, R., . . . Manni, G. (2008). The role of Humphrey Matrix testing in the early diagnosis of retinopathy in type 1 diabetes. British Journal of Ophthalmology, 92(12), 1656-1660. DOI: https://doi.org/10.1136/bjo.2008.143057.

* Pender, P. M., Benson, W. E., Compton, H. y Cox, G. B. (1981). The effects of panretinal photocoagulation on dark adaptation in diabetics with proliferative retinopathy. Ophthalmology, 88(7), 635-638.

- Petzold, G. C. y Murthy, V. N. (2011). Role of astrocytes in neurovascular coupling. Neuron, 71(5), 782-797. Dol: https://doi.org/10.1016/j.neuron.2011.08.009. 
* Reijmer, Y. D., Brundel, M., de Bresser, J., Kappelle, L. J., Leemans, A., Biessels, G. J. y Utrecht Vascular Cognitive Impairment Study, G. (2013). Microstructural white matter abnormalities and cognitive functioning in type 2 diabetes: a diffusion tensor imaging study. Diabetes Care, 36(1), 137-144. Dol: https://doi.org/10.2337/ dc12-0493.

Runkle, E. A. y Antonetti, D. A. (2011). The blood-retinal barrier: structure and functional significance. Methods in Molecular Biology, 686, 133-148. Dol: https://doi. org/10.1007/978-1-60761-938-3 5.

Russell, P. W., Sekuler, R. y Fetkenhour, C. (1985). Visual function after pan-retinal photocoagulation: a survey. Diabetes Care, 8(1), 57-63.

* Saydah, S. H., Fradkin, J. y Cowie, C. C. (2004). Poor control of risk factors for vascular disease among adults with previously diagnosed diabetes. JAMA, 291(3), 335-342. DOI: https://doi.org/10.1001/jama.291.3.335.

Schafer, D. P., Lehrman, E. K., Kautzman, A. G., Koyama, R., Mardinly, A. R., Yamasaki, R., ... Stevens, B. (2012). Microglia sculpt postnatal neural circuits in an activity and complement-dependent manner. Neuron, 74(4), 691-705. Dol: https://doi. org/10.1016/j.neuron.2012.03.026.

- Seiberth, V., Alexandridis, E. y Feng, W. (1987). Function of the diabetic retina after panretinal argon laser coagulation. Graefes Archive Clinical and Experimental Ophthalmology, 225(6), 385-390.

- Simo, R., Hernandez, C. y European Consortium for the Early Treatment of Diabetic, R. (2012). Neurodegeneration is an early event in diabetic retinopathy: therapeutic implications. British Journal of Ophthalmology, 96(10), 1285-1290. Dol: https://doi. org/10.1136/bjophthalmol-2012-302005.

- Sinclair, S. H., Grunwald, J. E., Riva, C. E., Braunstein, S. N., Nichols, C. W. y Schwartz, S. S. (1982). Retinal vascular autoregulation in diabetes mellitus. Ophthalmology, 89(7), 748-750.

- Stem, M. S. y Gardner, T. W. (2013). Neurodegeneration in the pathogenesis of diabetic retinopathy: molecular mechanisms and therapeutic implications. Current Medical Chemistry, 20(26), 3241-3250.

- Umegaki, H. (2012). Neurodegeneration in diabetes mellitus. Advance in Experimental Medical Biology, 724, 258-265. Dol: https://doi.org/10.1007/978-1-4614-0653-2 19.

* van Dijk, H. W., Verbraak, F. D., Kok, P. H., Garvin, M. K., Sonka, M., Lee, K., . . Abramoff, M. D. (2010). Decreased retinal ganglion cell layer thickness in patients with type 1 diabetes. Investigation in Ophthalmology and Vision Science, 51(7), 3660 3665. Dol: https://doi.org/10.1167/iovs.09-5041.

Volgyi, B., Kovacs-Oller, T., Atlasz, T., Wilhelm, M. y Gabriel, R. (2013). Gap junctional coupling in the vertebrate retina: variations on one theme? Progress in Retina and Eye Research, 34, 1-18. Dol: https://doi.org/10.1016/j.preteyeres.2012.12.002.

Wilkinson, C. P., Ferris, F. L., 3rd, Klein, R. E., Lee, P. P., Agardh, C. D., Davis, M., .. Global Diabetic Retinopathy Project, G. (2003). Proposed international clinical diabetic 
retinopathy and diabetic macular edema disease severity scales. Ophthalmology, 110(9), 1677-1682. Dol: https://doi.org/10.1016/S0161-6420(03)00475-5.

* Wolter, J. R. (1961). Diabetic retinopathy. American Journal of Ophtalmology, 51, 1123-1141.

Wood, J. R., Miller, K. M., Maahs, D. M., Beck, R. W., Dimeglio, L. A., Libman, I. M., ... Woerner, S. E. (2013). Most Youth With Type 2 Diabetes in the T1D Exchange Clinic Registry Do Not Meet American Diabetes Association or International Society for Pediatric and Adolescent Diabetes Clinical Guidelines. Diabetes Care, 36(7): 20352037. Dol: https://doi.org/10.2337/dc12-1959.

* Zlokovic, B. V. (2008). The blood-brain barrier in health and chronic neurodegenerative disorders. Neuron, 57(2), 178-201. Dol: https://doi.org/10.1016/j. neuron.2008.01.003.

\section{Cómo citar este artículo}

* Thébault, Stéphanie (2019). Pérdida de visión por diabetes: detección y tratamiento. Revista Digital Universitaria (RDU). Vol. 20, núm. 3 mayo-junio. Dol: http://doi.org/10.22201/codeic.16076079e.2019.v20n3.a1.

Recepción: 05/01/18. Aprobación: 23/11/18. 